\title{
Altered trigeminal system excitability in menstrual migraine patients
}

\author{
Ayhan Varlibas $\cdot$ A. Kemal Erdemoglu
}

Received: 29 November 2008/Accepted: 13 May 2009/Published online: 4 June 2009

(C) Springer-Verlag 2009

\begin{abstract}
To evaluate brainstem excitability in menstrual migraine (MM) patients and compare the electrophysiological parameters of the trigeminocervical reflex (TCR) during the perimenstrual (headache period) and follicular (headache-free) periods with those in healthy controls. Thirty-one patients with MM and 22 volunteer age- and sexmatched healthy women were included in the study. The TCR was studied bilaterally with stimulation of the supraorbital branch of the trigeminal nerve during the perimenstrual period and follicular phase. The electrophysiological parameters of the TCR were compared between MM patients and controls. In controls, there was no statistically significant difference in the mean reflex latencies recorded during the perimenstrual and follicular phases $(P>0.05)$. In MM patients, the mean reflex latencies recorded during the perimenstrual (headache period) and follicular phase (headache-free) periods were significantly different from each other and from those in controls. The latencies of MM patients during the follicular (headache-free) period were significantly longer than those of controls. Brainstem excitability differed significantly between the perimenstrual (headache period) and follicular phase (headache-free) periods in MM. Furthermore, trigeminal excitability in MM patients was significantly different from that in healthy controls in both phases of the menstrual period.
\end{abstract}

Keywords Menstrual migraine . Trigeminocervical reflex $\cdot$ Excitability $\cdot$ Trigeminal . Electrophysiology

\footnotetext{
A. Varlibas · A. K. Erdemoglu ( $₫)$

Department of Neurology, Faculty of Medicine,

Kirikkale University, 07100 Kirikkale, Turkey

e-mail: akerdemoglu@yahoo.com
}

\section{Introduction}

Neurophysiological studies are performed in order to explore and understand the pathophysiological mechanisms and the primary role of trigeminal system in migraine and other primary headaches [1-3]. Despite the advanced imaging methods, the excitability of neuronal structures of the brainstem in migraine and other primary headaches is determined by increasing number of neurophysiologic studies such as the trigeminofacial and TCRs in the recent years. The TCR, first defined in 1987 by Sartucci et al. [4], was suggested as a suitable method to study the excitability of trigeminal system. TCR is a nociceptive reflex which provides an opportunity for evaluation of activation changes of the area between trigeminocaudal nucleus and dorsal cervical spinal horn. Although the central pathways for the TCR in humans are unknown, they were supposed to be confined to the rostral portion of the spinal trigeminal nucleus (nucleus oralis) and projecting medially and ventrally to the spinal nucleus of the accessory nerve on both sides [5, 6]. The early responses of the TCR are probably disynaptic or oligosynaptic and mediated by non-nociceptive afferents functionally resembling the R1 component of the blink reflex [7, 8]. However, the late responses of the TCR are nociceptive inputs probably transmitted through a polysynaptic route, including the spinal trigeminal nuclei and reaching the cervical motor neurons [5, 9-11].

In the literature, there are only few reports about the TCR in migraine [12-15]. Moreover, no studies are reported on TCR investigation of MM patients. The aim of this study was to evaluate trigeminal nerve and brainstem excitability in MM patients and compare the electrophysiological parameters of the TCR during the perimenstrual and follicular periods with those in healthy-controls. 


\section{Patients and methods}

Thirty-one patients with perimenstrual migraine and 22 volunteer age- and sex-matched healthy women as controls were included into the study. The local ethics committee of the university hospital approved the study. Each participant gave written informed consent to participate in the study.

Patients were diagnosed with MM (pure MM or menstrual related migraine) according to the last International Headache Society classification [15]. IHS defines pure MM as the headache attack for migraine occurs exclusively on day $1 \pm 2$ of menstruation in at least two out of three menstrual cycles and at no other times of the cycle; menstrually related migraine as headache occurs on day $1 \pm 2$ of menstruation in at least two out of three menstrual cycles and additionally at other times of the cycle. The detailed history and clinical examination were performed prior to the study. Patients with structural lesions of the brain and other local or systemic diseases causing headache were excluded. MM patients and controls were evaluated for concomitant diseases through detailed history and neurological examination. In this study, the term MM covers both pure $\mathrm{MM}$ and menstrually related migraine. All selected patients had been suffering from migraine attacks for a minimum period of 3 years, with at least two migraine attacks in a month. The migraine group was asked to keep an event log for the last 3 months prior to the study and record the number of days with headache and severity of the attacks. The severity of pain was scored on a scale of 5. The patient and the control group did not use any prophylactic medication.

The exclusion criteria were:

1. Younger than 18 or older than 45 years,

2. Migraine headaches that do not fulfill the IHS 2004 criteria,

3. A current history of alcohol addiction or drug abuse including acute headache medications. Drug abuse was defined according to the criteria proposed by the HIS,

4. Irregular menstrual periods, amenorrhea, hormone replacement therapy or other hormonal treatment including oral contraceptives within 6 months prior to the start of the study or at any time during the study period,

5. Lactating, pregnant, or intending to become pregnant during the study,

6. Suffering from any substantial systemic, neurological or psychiatric disease. History of cardiac, renal or liver disease or significantly abnormal EKG, liver or renal function tests.
Electrophysiological method

In electrophysiological study, TCR was obtained bilateral using Dantec Key-Point 4C. The stimulation was applied to the supraorbital branch of the trigeminal nerve via surface electrodes. The electromyographic activity was recorded bilaterally, by means of surface electrodes, from symmetrical sites on the upper half of each resting sternocleidomastoid muscle. The pair of surface electrodes was positioned $3 \mathrm{~cm}$ apart in a longitudinal direction over the muscles as described previously [9]. Electrical stimuli with $0.1 \mathrm{~ms}$ duration were applied bilaterally to the supraorbital trigeminal branch near the point of nerve exit from the skull. The intensity was adjusted to reach three times the perception threshold and was felt to be strong but not painful. Three to seven consecutive responses were averaged in each trace. Bilateral, one-component reflex responses were obtained regularly from SCM tested at rest as described previously [9, 12].

The analysis time was $100-200 \mathrm{~ms}$, and the filter bandpass was $20 \mathrm{~Hz}$ to $3 \mathrm{kHz}$. Sensitivity was set at 200$500 \mu \mathrm{V}$. During the study, room temperature was maintained between 22 and $24^{\circ} \mathrm{C}$. The onset latency (ms), area $(\mathrm{mV} \times \mathrm{ms})$ and peak-to-peak amplitude $(\mathrm{mV})$ of the reflex responses were recorded during the perimenstrual period and in the follicular phase in each group. The onset of long latency was termed as $\mathrm{R}$ and contralateral side as CR.

The trigemino-cervical reflex was recorded during perimenstrual period or menstrual phase (headache period, in 4-6 h of the migraine attack) and postmenstrual follicular phase, the headache-free period in MM patients. Control patients were also evaluated at the same time of menstrual periods with MM patients. The results were compared between migraine patients and controls and between perimenstrual and postmenstrual follicular periods of each group. The recordings were performed by the same researcher who was blinded, at the time of the electrophysiological study, as to the status and period of the subject.

Statistical analyses

All the data (categorical and numerical) of MM patients and controls recorded during perimenstrual headache period, postmenstrual follicular period were evaluated by SPSS for Windows 9.0 (Chicago, IL, USA version) program. The mean values of the groups were compared by the paired $t$ test, Student's $t$ test or Mann-Whitney rank sum test. $P$ value $<0.05$ was accepted as statistically significant. 
Table 1 Clinical characteristics of the patients with menstrual migraine

\begin{tabular}{ll}
\hline Mean duration of migraine attack & $28.7 \pm 15.3 \mathrm{~h}(6-56)$ \\
Age of migraine (months) & $47.48 \pm 14.81$ \\
Attack frequency per month & $2.33 \pm 0.75(1-3)$ \\
Mean severity (over 5) & $4.19 \pm 0.74(3-5)$ \\
Localization of the pain & \\
Unilateral & 27 \\
Right-sided predominantly & 13 \\
Left-sided predominantly & 14 \\
Bilateral & 4 \\
Aura (+) & 8 \\
Family history of migraine $(+)$ & 13 \\
Pure menstrual migraine & 3 \\
\hline
\end{tabular}

\section{Results}

The study group consisted of 31 patients with MM and 22 healthy control subjects at similar ages. The mean age of the MM patients was $34.9 \pm 6.5$, ranging between 20 and 45 years. The mean age of the control subjects was $32.5 \pm 7.2$, ranging between 19 and 45 years $(P>0.05)$. The clinical features of $\mathrm{MM}$ patients are presented in Table 1.

As TCR recordings were made bilaterally from SCM at rest, early responses were not elicited. In controls, the mean latencies of bilateral $\mathrm{R}$ and $\mathrm{CR}$ of TCR recorded during the perimenstrual and follicular phase did not change significantly $(P>0.05)$ but in MM patients there was a significant difference in the mean latencies of bilateral R and CR of TCR between headache and headache-free period (Fig. 1). However, the mean long latencies of TCR in MM patients were significantly different from those of healthy controls. Interestingly, the latencies of MM patients during follicular phase were significantly longer than those of controls (Table 2).

The TCR mean amplitudes and mean areas did not change between the perimenstrual or postmenstrual follicular periods of MM patients and controls $(P>0.05)$. The TCR results were evaluated according to the perimenstrual clinical findings of MM patients. When the mean latencies of TCR of the patients were evaluated according to localization of the headache, presence of family history and aura, age of migraine, headache intensity, frequency and duration, no statistically significant difference was found in the mean latencies of TCR $(P>0.05)$ (Table 3).

\section{Discussion}

In our study, the long latencies, amplitudes and area of TCR were evaluated in different phases of MM patients
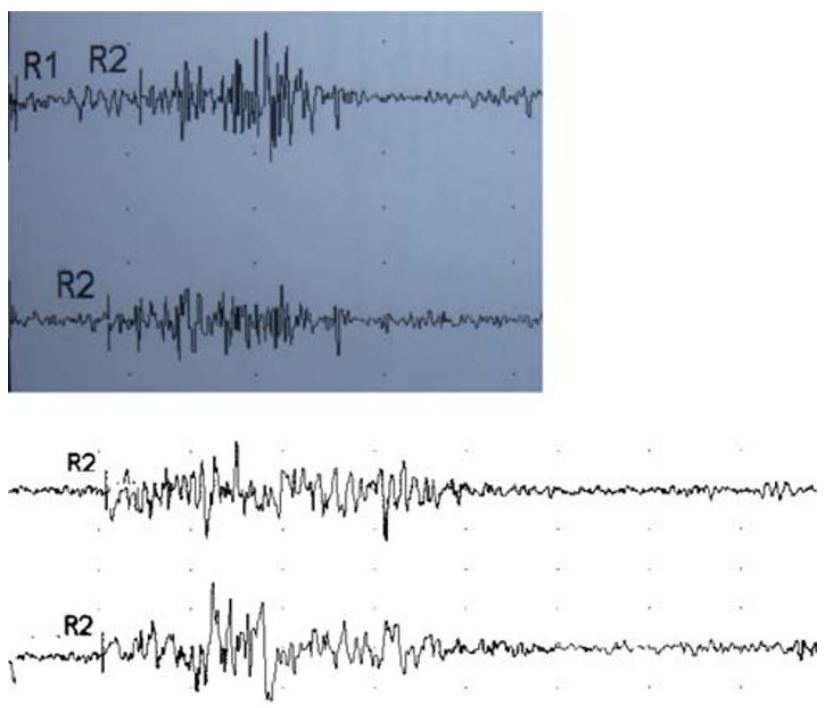

Fig. 1 Trigemino-cervical reflex in menstrual migraine patient (upper trace) and control group (lower trace)

and controls. It was found that the long latency of TCR was of shortened latency in all patients with headache, compared with headache-free and the controls. During migraine attack, altered trigeminal nociception was shown in clinical studies and hyperexcitability has been suggested in brainstem neurophysiologic studies in migraine patients. Recently, several studies on the nociceptive blink reflex have shown a sensitization phenomenon of the trigeminal pathways in migraine patients during the attack [17-19]. In recent years, there are a few studies obtained shorter duration of short latencies of TCR performed during headache and headache-free. Nardone et al. [13] studied migraine patients during headache-free and headache period and found that the trigeminocervical responses were bilaterally abnormal in 17 patients with migraine with aura and 15 patients with migraine without aura during the headache attacks, in 11 patients with migraine with aura and in 10 patients with migraine without aura during the interictal period. It was reported that the results were indicative of abnormalities of the interneuronal brainstem pathways mediating the oligosynaptic TCR in migraine patients during the headache attacks and during the interictal phase [13]. Milano et al. [12] studied long latency of TCR of migraine patients during headache-free period and found that the mean onset TCR after ipsi- and contralateral stimulation was significantly shortened on the painful side of migraine patients $(P<0.01)$. Recently, the recovery curve of the TCR was found to be significantly faster in migraine patients than in controls, suggesting that the painfree period in migraine patients was characterized by a hyperexcitability of the trigeminal pathways [14]. In our study, the long latency of TCR was of shortened latency in 
Table 2 Note the statistical differences in the mean latencies of menstrual migraine patients during the perimenstrual and follicular phases were significantly different from each other $(P<0.01)$ and from those of controls $(P<0.05)$

\begin{tabular}{|c|c|c|c|c|c|c|}
\hline & \multicolumn{2}{|l|}{ Menstrual migraine } & \multirow[t]{2}{*}{$P$ value } & \multicolumn{2}{|l|}{ Controls } & \multirow[t]{2}{*}{$P$ value } \\
\hline & $\begin{array}{l}\text { Perimenstrual period } \\
\text { (headache attack) }\end{array}$ & $\begin{array}{l}\text { Postmenstrual follicular } \\
\text { period (headache-free) }\end{array}$ & & $\begin{array}{l}\text { Perimenstrual } \\
\text { period }\end{array}$ & $\begin{array}{l}\text { Postmenstrual } \\
\text { follicular period }\end{array}$ & \\
\hline Right side $\mathrm{R}$ & $32.50 \pm 3.52$ & $43.90 \pm 4.02$ & $P<0.01$ & $40.57 \pm 2.77$ & $40.92 \pm 2.92$ & $P>0.05$ \\
\hline Right side CR & $32.75 \pm 3.34$ & $44.40 \pm 4.30$ & $P<0.01$ & $40.74 \pm 2.80$ & $41.28 \pm 2.92$ & $P>0.05$ \\
\hline Left side $R$ & $32.68 \pm 3.50$ & $43.91 \pm 3.79$ & $P<0.01$ & $40.63 \pm 2.65$ & $40.47 \pm 2.94$ & $P>0.05$ \\
\hline Left side CR & $32.93 \pm 3.40$ & $44.35 \pm 4.03$ & $P<0.01$ & $41.02 \pm 2.70$ & $40.78 \pm 3.05$ & $P>0.05$ \\
\hline
\end{tabular}

There was no statistical difference between menstrual periods in controls $(P>0.05)$. Values are expressed as $\mathrm{ms}$

$R$ right, $L$ left, $R 1$ perimenstrual $\mathrm{R}, C R$ contralateral, $R 2$ postmenstrual follicular

Table 3 The mean amplitudes and area under the curve of the reflex responses in menstrual migraine patients and controls recorded during the perimenstrual and postmenstrual follicular period

\begin{tabular}{|c|c|c|c|c|c|c|}
\hline & \multicolumn{2}{|l|}{ Menstrual migraine } & \multirow[t]{2}{*}{$P$ value } & \multicolumn{2}{|l|}{ Control } & \multirow[t]{2}{*}{$P$ value } \\
\hline & $\begin{array}{l}\text { Perimenstrual period } \\
\text { (headache attack) }\end{array}$ & $\begin{array}{l}\text { Postmenstrual follicular } \\
\text { period (headache-free) }\end{array}$ & & $\begin{array}{l}\text { Perimenstrual } \\
\text { period }\end{array}$ & $\begin{array}{l}\text { Postmenstrual } \\
\text { follicular period }\end{array}$ & \\
\hline \multicolumn{7}{|l|}{ Area $(\mathrm{mV} \times \mathrm{ms})$} \\
\hline Right side area $\mathrm{R}$ & $3.6 \pm 1.63$ & $4.55 \pm 1.41$ & 0.06 & $3.86 \pm 2.35$ & $3.86 \pm 2.33$ & 0.98 \\
\hline Right side area CR & $3.81 \pm 1.47$ & $4.41 \pm 1.54$ & 0.20 & $3.51 \pm 2.26$ & $3.84 \pm 2.34$ & 0.88 \\
\hline Left side area $\mathrm{R}$ & $4.01 \pm 1.93$ & $4.71 \pm 1.38$ & 0.23 & $4.06 \pm 2.56$ & $3.79 \pm 2.31$ & 0.58 \\
\hline Left side area CR & $3.99 \pm 1.99$ & $4.83 \pm 1.52$ & 0.16 & $3.72 \pm 2.38$ & $3.54 \pm 2.20$ & 0.61 \\
\hline \multicolumn{7}{|c|}{ Amplitude (AM) (mV) } \\
\hline Right side AM R & $0.43 \pm 0.19$ & $0.47 \pm 0.18$ & 0.27 & $0.44 \pm 0.21$ & $0.45 \pm 0.29$ & 0.89 \\
\hline Right side AM CR & $0.69 \pm 0.94$ & $0.48 \pm 0.23$ & 0.34 & $0.45 \pm 0.29$ & $0.44 \pm 0.25$ & 0.81 \\
\hline Left Side AM R & $0.48 \pm 0.32$ & $0.46 \pm 0.17$ & 0.70 & $0.40 \pm 0.19$ & $0.47 \pm 0.29$ & 0.30 \\
\hline Left side AM CR & $0.48 \pm 0.19$ & $0.50 \pm 0.24$ & 0.55 & $0.45 \pm 0.29$ & $0.45 \pm 0.28$ & 0.91 \\
\hline
\end{tabular}

Values are expressed as $\mathrm{mV}$ and $\mathrm{mV} \times \mathrm{ms}$, for amplitude and area, respectively

all patients with headache during the perimenstrual period, compared with headache-free and the controls $(P<0.01)$.

Our results are compatible and consistent with the previous studies showing the increased excitability of trigeminal system during migraine attack. However, there is a considerable difference in our results with previous studies showing the trigeminal hyperexcitability in headache-free period, though none of them studied MM patients. In previous studies, it was suggested that trigeminal hyperexcitability was present in headache-free period. In a literature review, we did not find any study investigating the trigeminal excitability of MM patients. In our study, although there was a significant difference in the excitability of trigeminal and brainstem neurons between perimenstrual period and follicular period in MM, suggesting that the excitability of trigeminal system did not increased during headache-free period when it was compared with the headache period and controls, even though the excitability of trigeminal system seems to be decreased. Besides these, although it was found that trigeminal excitability of MM patients was significantly different from controls, there was not any difference in the variability of trigeminal excitability in controls between the perimenstrual period and follicular period. Hence, the alteration in the excitability of MM patients may not be attributed only to hormonal changes during menstrual period.

The results of the controls were similar to those previously reported studies [9, 11-14]. However, the effect of hormonal change on with neurophysiologic studies of trigeminal system of controls has not been studied extensively so far. Although hormonal changes have been reported to have an indirect influence of brainstem nociceptive modulatory system in tension-type headache, we did not found any significant difference between the perimenstrual and postmenstrual follicular period of healthy volunteers with respect to onset latency of TCR [16]. In our study, these results showed that trigeminal excitability did not change significantly during the different menstrual periods of controls.

Besides all, no significant differences were found in the mean latencies of the TCR of the MM patients recorded during the perimenstrual period and postmenstrual follicular period when compared according to the parameters of 
presence aura and family history, mean duration and number of attacks per month and location of headache $(P>0.5)$. Milanov et al. [13] showed activity differences between the brainstem interneurons of the painful side and pain-free side obtained by TCR of migraine patients with unilateral headache. In our study, we did not observe activity changes between the interneurons of the two sides of the brainstem in patients with unilateral and bilateral headaches. The activation changes between the brainstem areas due to location of pain may suggest that activation changes during the pain attacks may affect the contralateral brainstem.

These results implying altered trigeminal excitability of the trigeminal system of MM in both headache and headache-free period and the brainstem excitability of MM patients was found to be different from that of normal subjects. However, it is not obvious whether the changes observed in trigeminal reflexes or brainstem during the headache and headache-free periods are related to change in the sensitivity of the trigeminal pathways or to change in the control of descending inhibitory modulation but the results are implying the impairment of pain control systems at the trigeminal level.

Although the exact pathophysiology of the $\mathrm{MM}$ is unknown, hormonal changes are thought to be responsible. While menstruation seems to be the primary or only trigger for migraine in $60 \%$ of women migraine patients, onset of menses or change in hormonal level does not appear to affect migraine frequency in the remaining $40 \%$ [20, 21]. Although the exact mechanism for MM remains unknown and hormonal change were supposed to be the main trigger for migraine attack of MM, there may be other accompanying factors besides abrupt hormonal drop, such as $\mathrm{Mg}$, genetic predisposition, gene regulations and expression, sensitivity of specific serotonin receptors, expression of neurotransmitters or peptides involved in nociception and prostaglandin release [22-26]. Welch et al. [25] proposed that estrogen modulates neuronal function resulting in a mismatch between its gene regulation and membrane effects in MM patients. Furthermore, it may be also supposed that the trigeminal excitability of MM patients may be different from migraine with or without aura patients. Central sensitization, which proposes that altered processing of sensory input in the brainstem, principally the trigeminal nucleus caudalis may play a critical role in menstrual migraine pathogenesis [27]. A limitation of our study is the relatively small number of MM patients and other primary headache disorders are not included in order to compare with those MM. Thus, further studies are needed to clarify this point.

\section{Conflict of interest None.}

\section{References}

1. Moskowitz MA (1992) Trigeminovascular system. Cephalalgia 12(3): 127

2. Bolay H, Reuter U, Dunn AK, Huang Z, Boas DA, Moskowitz MA (2002) Intrinsic brain activity triggers trigeminal meningeal afferents in a migraine model. Nat Med 8(2):136-142

3. Welch KM (2003) Contemporary concepts of migraine pathogenesis. Neurology 61(8 Suppl 4):S2-S8

4. Sartucci F, Rossi A, Rossi B (1986) Trigemino cervical reflex in man. Electromyogr Clin Neurophysiol 26(2):123-129

5. Astelmark B, Pinter MJ, Sasaki S, Tantisira B (1992) Trigeminal excitation of dorsal neck motoneurones in the cat. Exp Brain Res 92:183-193

6. Kato S, Papuashvili N, Okada YC (2003) Identification and functional characterization of the trigeminal ventral cervical reflex pathway in the swine. Clin Neurophysiol 114:263-271

7. Di Lazzaro V, Quartarone A, Higuchi K, Rothwell JC (1995) Short-latency trigemino-cervical reflexes in man. Exp Brain Res 102(3):474-482

8. Di Lazzaro V, Restuccia D, Nardone R et al (1996) Preliminary clinical observations on a new trigeminal reflex: the trigeminocervical reflex. Neurology 46:479-485

9. Milanov I, Bogdanova D, Ishpekova B (2001) The trigeminocervical reflex in normal subjects. Funct Neurol 16(2):129-134

10. Ertekin C, Celebisoy N, Uludag B (1996) Trigemino-cervical reflexes in normal subjects. J Neurol Sci 143:84-90

11. Serrao M, Rossi P, Parisi L, Perrotta A, Bartolo M, Cardinali P, Amabile G, Pierelli F (2003) Trigemino-cervical-spinal reflexes in humans. Clin Neurophysiol 114(9):1697-1703

12. Headache Classification Subcommittee of the International Headache Society (2004) International classification of headache disorders. Cephalalgia 24(Suppl 1):1-152

13. Milanov I, Bogdanova D (2003) Trigemino-cervical reflex in patients with headache. Cephalalgia 23(1):35-38

14. Nardone R, Tezzon F (2003) Short latency trigemino-sternocleidomastoid response in patients with migraine. J Neurol 250 (6):725-732

15. Serrao M, Perrotta A, Bartolo M, Fiermonte G, Pauri F, Rossi P, Parisi L, Pierelli F (2005) Enhanced trigemino-cervical-spinal reflex recovery cycle in pain-free migraineurs. Headache $45(8)$ : 1061-1068

16. Schoenen J, Bottin D, Sulon J, Gaspard U, Lambotte R (1991) Exteroceptive silent period of temporalis muscle in menstrual headaches. Cephalalgia 11(2):87-91

17. Kaube H, Katsarava Z, Przywara S, Drepper J, Ellrich J, Diener HC (2002) Acute migraine headache: possible sensitization of neurons in the spinal trigeminal nucleus? Neurology 58(8):12341238

18. Katsarava Z, Lehnerdt G, Duda B, Ellrich J, Diener HC, Kaube H (2002) Sensitization of trigeminal nociception specific for migraine but not pain of sinusitis. Neurology 59(9):1450-1453

19. De Marinis M, Pujia A, Colaizzo E, Accornero N (2007) The blink reflex in "chronic migraine". Clin Neurophysiol 118(2): 457-463

20. Stewart WF, Lipton RB, Celentano DD, Reed ML (1992) Prevalence of migraine headache in the United States. Relation to age, income, race, and other sociodemographic factors. JAMA 267(1): 64-69

21. Loder EW (2006) Menstrual migraine: pathophysiology, diagnosis, and impact. Headache 46(Suppl 2):S55-S60

22. Puri V, Cui L, Liverman CS, Roby KF, Klein RM, Welch KM, Berman NE (2005) Ovarian steroids regulate neuropeptides in the trigeminal ganglion. Neuropeptides 39(4):409-417 
23. Martin VT, Behbehani M (2006) Ovarian hormones and migraine headache: understanding mechanisms and pathogenesis-part I. Headache 46(1):3-23

24. Zacur HA (2006) Hormonal changes throughout life in women. Headache 46(Suppl 2):S49-S54

25. Welch KM, Brandes JL, Berman NE (2006) Mismatch in how oestrogen modulates molecular and neuronal function may explain menstrual migraine. Neurol Sci 27(Suppl 2):S190-S192
26. Mauskop A, Altura BT, Altura BM (2002) Serum ionized magnesium levels and serum ionized calcium/ionized magnesium ratios in women with menstrual migraine. Headache 42(4):242248

27. Dodick D, Silberstein S (2006) Central sensitization theory of migraine: clinical implications. Headache 46(Suppl 4):S182S191 